frequently in some developed countries about HIV management. HIV is a public health concern in all spheres of health care and its guidelines need to be constantly updated. The objective is to present the development of the virtual strategies for improvement of quality of health of HIV care in Latin America community-based Primary Care (PC) practices.

Methods A descriptive study of the development of a learning object was carried out to improve effectiveness of care of HIV by the PC physicians. The structure and elements of a traditional $\mathrm{AD}$ strategy were adapted by an educational outreach program.

Results The development of a learning object led to the compilation of materials necessary for the professional practice of HIV treatment, including models for notification, prescription, segmental examinations and the development of a flow for decision making, including multidisciplinary aspects such as contraception, social benefits, control of opportunistic infections, and others. The instructional design was structured to facilitate navigation, and can be used as support material during consultations, and can be easily accessed by mobile or computers.

Conclusion After the START and TEMPRANO studies trials, which are tools extensively used in the management of HIV, PC practices assume a pivotal part of the care of people living with HIV. The Guidellines' priority agenda of updating includes a number of other chronic and acute illnesses, however, especially in developing countries, there is no sheltered schedule for the physician to undertake lifelong education that includes so many updates. Virtual $\mathrm{AD}$ has the potential to reach out a large number of physicians, even with difficult access or time, without losing the one-by-one training feature. Disclosure No significant relationships.

\section{P181 EPIDEMIOLOGICAL INFORMATION ON HIV IN THE MANAGEMENT OF THE CASCADE OF CARE IN HEALTH SERVICES}

${ }^{1}$ Ana Amélia Bones, ${ }^{2}$ Karla Viana, ${ }^{1}$ Silvio Cazella, ${ }^{3}$ Airton Stein. ${ }^{1}$ Health Federal University from Porto Alegre, Health Informatics Post Graduation Program, Porto Alegre, Brazil; ${ }^{2}$ Federal University of Rio Grande do Sul, Porto Alegre, Brazil; ${ }^{3}$ Health Federal University from Porto Alegre, Health Science Post Graduation Program, Porto Alegre, Brazil

\subsection{6/sextrans-2019-sti.336}

Background According to UNAIDS, an estimated 100,000 cases of new HIV infections were reported in Latin America in 2017. At the time, Antiretroviral Therapy (ART) was already being offered for these new cases, and was largely prescribed in the Primary Health Care (PHC) system. The objective of this study is to evaluate the epidemiological profile of new cases of HIV infection in a Porto Alegre City, with some of the worst HIV indicators in Latin America.

Methods A cross-sectional study, was carried out using a time series analysis, with data from Brazilian Information Systems. Data mining with WEKA software had been applied to evaluate the variables. The inclusion criteria were HIV diagnosis in the 2017 year, over 13 years who live in Porto Alegre City.

Results The incidence of HIV cases was 777 in Porto Alegre, in which the population is 1.500 .000 inhabitants. There were $308(39.6 \%)$ women, 71(34.8\%) blacks and mulattoes and 40 $(5.1 \%)$ were pregnant. Of these, $60(7.7 \%)$ presented viral load above 100,000 copies/mL and 267(34.3\%) below 10,000
copies/mL. The number of people with CD4 count below 200 was $26(3.3 \%)$ and $455(58.5 \%)$ people had a time interval shorter than 3 months between the diagnosis and the beginning of ART. ARTs with Tenofovir/Lamivudine/Dolutegravir and Tenofovir/Lamivudine/Efavirenz were prescribed in 566 (95\%) of the 590 patients who started ART. Among the 777 cases, 521(67\%) were diagnosed through the Rapid Test, which are offered primarily in PHC.

Conclusion The role of PHC was seen to be effective in reaching 90-90-90 Targets, since it has increased the diagnosis of HIV to immunocompetent individuals, facilitating easy access to first-line ART in short period of time. Future studies should be designed to investigate the role of PHC in the cascade of care for individuals with HIV. Information obtained from data mining can be applied to organize strategic interventions focused on local realities.

Disclosure No significant relationships.

\section{P183 PREDICTORS OF CHLAMYDIA TRACHOMATIS AND NEISSERIA GONORRHOEAE ASYMPTOMATIC INFECTION IN HIV PATIENTS}

Luísa Graça, Isabel Gomes Abreu, Joana Sobrinho Simões, Nuno Rocha Pereira, Carmela Piñeiro, Jorge Soares, Rosário Serrão, Cátia Caldas*, António Sarmento. Centro Hospitalar e Universitário de São João, Infectious Diseases, Porto, Portugal

\subsection{6/sextrans-2019-sti.337}

Background The prevalence of sexually transmitted infections (STIs), namely Chlamydia trachomatis (CT) and Neisseria gonorrhoeae (NG), is rising, possibly as a consequence of the adoption of risky sexual behaviour as HIV infection prognosis improved. Most STIs are asymptomatic. Accordingly, treating only symptomatic patients has only a minor impact on the prevalence of such infections. This study aims to identify risk factors for asymptomatic CT and NG infection in a population of patients with HIV infection.

Methods We retrospectively evaluated the clinical records of 218 adult patients with HIV infection who were screened for NG and CT infection from July 2017 to June 2018 in a tertiary care teaching hospital. Urine, rectal, oropharyngeal and vaginal samples were tested by nucleic acid amplification test (NAAT). The association of independent variables with STIs occurrence was evaluated using logistic regression.

Results We identified 45 cases of STIs (25 NG, 20 CT), 20.64\%. All patients were male. Although 95.6\% of identified infections were extragenital, 96 (44\%) patients collected only genital samples. In univariate analysis, STI was significantly associated with younger age, being a men who has sex with men (MSM), a higher CD4+ T cell count, having classic symptoms of STI in another location and a positive treponemic test. In a multivariate model being MSM (OR 5,011; 95\%CI 1,286 - 19,519; $\mathrm{p}=0,020)$ and having classic symptoms of STI in another location (OR 8,586; 95\%CI 3,342 $22,062 ; \mathrm{p}=0.00)$ were independent risk factors.

Conclusion Although HIV patients are a risky population, with a high prevalence of STIs, it is not homogeneous and so it matters to identify those who had classic symptoms of STI in another location. Screening only in genital samples will miss the majority of infections.

Disclosure No significant relationships. 